\title{
An Improved Minimum-Cost Pathfinding Algorithm for Mobile Robot Navigation
}

\author{
Yung-Fu Hsu, Chun-Hao Huang, Way-Ren Huang, and Woei-Chyn Chu
}

\begin{abstract}
In this paper, we present an effective pathfinding algorithm for mobile robot navigation. The algorithm stem is from the $A^{*}$ algorithm that can perform minimum-cost path navigation with the added obstacles avoidance capability. A set of passive RFID tags placed on the Cartesian grids where used for location identification. During navigation, the RFID reader attached on the mobile robot receives its nearby RFID tags' ID to determine the robot's current position, and use it to formulate the minimum-cost path that goes from the present location to the target point. The devised path is a set of connected RFID tags computed by the $\mathrm{A}^{*}$ algorithm. The proposed improved $A^{*}$ algorithm explored a heuristic search of the minimal cost navigation path that taking into consideration of the presence of obstacles so that the devised optimal path would not collide with the obstacles. Passive RFID tags have the advantages of low cost, providing unequivocal coordinate information, fast computation and easy deployment.
\end{abstract}

Index Terms-Pathfinding, A* algorithm, robot navigation, passive RFID.

\section{INTRODUCTION}

Mobile robots have been widely applied in the fields of path patrolling, environmental searching, objects tracking, tour guiding or even house cleaning, etc. The critical aspect of these applications is path-planning that validly converts complicated conditions into intelligibility. Unlike manual operation, artificially intelligent settings in pathfinding can automatically generate accessible paths by relied searching manners in defined space. In other words, the intention is to provide sufficiently navigating information and collision-free passages for achieving significant utility. However, the point-to-point problem is an issue among these, generally refers to guarantee a shortest route between two end points. In this kind of solutions, there were several famous approaches to deal with, such as Dijkstra's algorithm, bread first search algorithm and depth first search algorithm [1]-[3]. However, these algorithms encounter a rough problem which probably will excessively explore and accumulate plenty of searching breadth under unweighted situation.

In order to rapidly find shortest path and reduce searching breadth, a heuristic approach was proposed which called A* algorithm to determine minimum-cost path [4]. In comparison with above mentioned approaches in identical area, $A *$ showed superiority of less computation due to the heuristic function could greatly diminish breadth by taking

Manuscript received March 20, 2013; revised April 28, 2013.

The authors are with the National Yang Ming University, Beitou, Taipei 11221 Taiwan (e-mail: d49904010 @ym.edu.tw,jim90486@hotmail.com, g39604042@gmail.com,wchu@ym.edu.tw). travelling distance into consideration. Nevertheless, it had covert shortcoming that the planned route generated redundant turns so that caused time waste and energy consumption while operating in practical implementation [5]. Besides weight-led methods, modus operandi of graph searching is also a considerable way to scheme navigating path, such as Visibility Graph [6] and Voronoi Diagram [7]. These approaches could effectively construct an accessible pathway form current location to goal site that depended on characteristics of obstacles and map information. However, both were belonged to global path planning and maybe caused huge computation regardless of how close between starting point and goal.

In the field of pathfinding at present, the shortest and collision-free path is the essential component practicing in real environments. However, an intelligent pathfinding system provided with both features is not enough conditions for rapidly cruising and arriving at destination. In the example of redundant veers in proposed approach, it isn't adequate to bring beneficial effect. Therefore, in this paper we first employ passive RFID tags as landmarks for location identification and another purpose is reducing graph computation. Then the major pathfinding technique is applied for navigation which is planned out a suited path by integrating the well-known shortest path searching $\mathrm{A}^{*}$ algorithm with an obstacle analysis and avoidance scheme, such that the proposed improved $\mathrm{A}^{*}$ algorithm can not only generate a collision free route but obviously decrease redundant turns. During navigation, the RFID reader also can real-time recognize the current mobile robot's position via determination of nearby RFID tags' information.

The remainder of the paper is organized as follows. The literatures about RFID technology for assisting mobile robot navigation and related works of path planning are described in Section II. The system architecture is introduced in Section III, includes basic RFID introduction, RFID deployment and path planning approach. In Section IV, we demonstrate the results of our purposed system with simulation and experiments. Finally, conclusions and future works are described in Section V.

\section{RELATED WORKS}

Radio Frequency Identification (FRID) technology was deployed for assisting mobile robot navigation has been widely studied. Recent approaches in this field have revealed an increasing interests in the oncoming RFID technology as promising alternative technique due to ease of use, flexibility, and low cost. In general, RFID tags were referred as landmarks which captured signals by readers for 
determination of robot's position and direction. In assisting navigation, RFID grid was one of the most commonly used with localization-estimated algorithms for offering more accurate position [8]-[12]. Besides, the gird configuration was commonly adopted the deployment of Cartesian or triangular. On the other hand, RFID tags were attached to specific location instead of grid shape which be used for estimating mobile robot's navigation was an alternative approach [13]-[15]. These systems usually equipped with single antenna or a pair of antennas on mobile robot to improve its positioning accuracy which relied on received information analysis.

Since RFID had significant evidence on improving mobile robot's localization, the path-planning is regarded as the principle that guides mobile robot form start point to destination. Just as we have been described in Section I, we will state more relative approaches with regard to path-planning in this paragraph. Ferguson et al. [16] introduced Field $D^{*}$ algorithm that used linear interpolation during planning to compute approximate path cost estimates for arbitrary positions within each grid cell and generate paths with a series of continuous courses. This approach could deploy in non-uniform resolution grids which was allowed paths to traverse between any two points on adjacent grid cell edges, rather than their centers or corners. Nakamiya et al. [5] purposed a method defined as cost map, which was in accordance with base cost matrix to achieve the lowest power consumption route. The base cost matrix included eight directions for each cell and its critical factor determined on current car-robot's direction. Bhattacharya et al. [17] used the Voronoi diagram for path planning and smooth the path for better obstacle avoidance by iterative refinement. Zhou [18] first adopted Visibility Graph based on obstacle vertices and Dijstra's search to build a shortest path, then incorporated potential field to accomplish robot motion.

\section{SySTEM ARCHITECTURE}

This section we narrate several main components of our purposed system architecture that contains RFID system and pathfinding approach. The deployment of passive RFID pattern is considered as promotion of localization accuracy with respect to mobile robot's position during cruise. Beside, these landmarks are also corresponding to grid cells which provide their essential information for path-planning after knowing present location and destination, then the outcomes of post-computing will feedback to instruct mobile robocar's motion. The detailed architecture describes as follows.

\section{A. RFID System}

In General, RFID systems consist of intelligent tags, a reader, one or more antenna(s), and software such as drives and middleware. A tag can be classified as three types: passive, semi-passive and active. In consideration of cost, no maintenance and localization accuracy, we pick passive tags as our navigating landmarks that belong to low frequency level and transmitting/receiving dependency on inductive coupling between two circuits of tags and reader. A reader mostly plays a transponder mainly capture of tag's identification via radio waves and return these information to back-end application system. On the basis of different functionality, it can be distinguished as a reader or a reader/writer. An antenna is sometimes regarded as a separate part of RFID system. It works as transmitting device of radio wave that establishes communication between a reader and tags relying on mutual inductance. Our purposed RFID apparatuses adopt HBE-FRID-RX series form HANBACK ELECTRONICS, which can be compatibly equipped on robocar by manufacturing in the same corporation. The frequency band for the RFID system is $900 \mathrm{MHz}$. Fig. 1 shows the body with integration of RFID reader and mobile robocar.

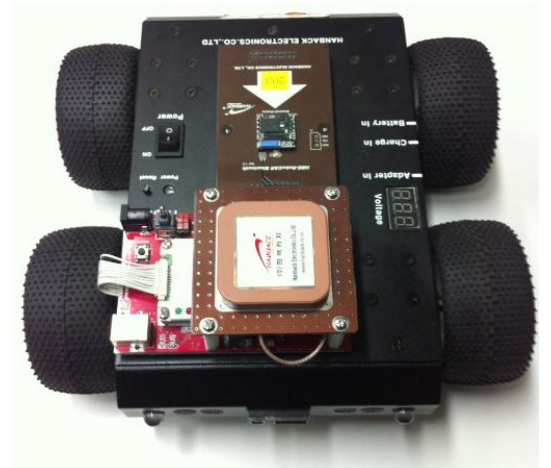

Fig. 1. The mobile robot was equipped with RFID reader and antenna, which manufactured by HANBACK ELECTRONICS.

\section{B. RFID-Based Localization Model}

In order to estimate mobile robot position using RFID system, passive tags are arranged as Cartesian shape in a fixed pattern on the floor, as shown in Fig. 2. The arranged tags are corresponded to real position information before path-planning and navigation. Each tag is given sequencing coordinate and stored in database for later exploitation. While mobile robot stays or cruises at someplace, the antenna generates an effective area that gathers these recognized tags, as shown in Fig 3. Thus, these recognized tags offer their unique serial numbers to transfer real coordinates, so that we can clearly acquire the mobile robot position. If the antenna detects multiple tags as $\operatorname{Tag} 1\left(x_{1}, y_{1}\right), \operatorname{Tag} 2\left(x_{2}, y_{2}\right) \ldots$ Tagn $\left(x_{n}, y_{n}\right)$, we can obtain the estimated coordinate by following questions, as (1) and (2).

$$
\begin{aligned}
& x_{\text {estimate }}=\frac{\left(x_{1}+x_{2}+\ldots+x_{n}\right)}{n} \\
& y_{\text {estimate }}=\frac{\left(y_{1}+y_{2}+\ldots+y_{n}\right)}{n}
\end{aligned}
$$

where $n$ is the detected numbers by antenna in the effective area and $\left(x_{i}, y_{i}\right), i=1,2, \ldots, n$ are belonging to those coordinates. However, in the case only single tag is recognized, the moment coordinate is rightly the mobile robot position. We know how the mobile robot gets its location by RFID pattern, next we figure out a minimum-cost path for mobile robot navigation. In addition, the existence of obstacle is the considerable situation due to there is collision occurrence if no any mechanism to prevent it, likes a scenario of Fig. 2. Therefore, we set up those tags which are pinned under the obstacles are recorded as disabled marks. The given information with regard to grid-like map can support to establish a collision-free path. 


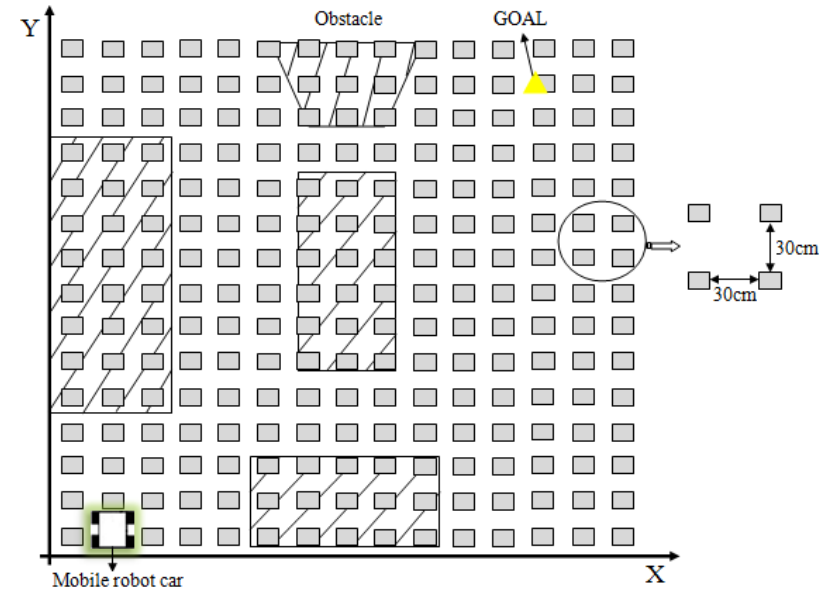

Fig. 2. The deployment of a set of passive RFID tags in a Cartesian grid. The initial position of the mobile robot is on the low-left corner while the destination target in marked by the yellow triangle on the top-right corner. Shaded areas indicate obstacles.

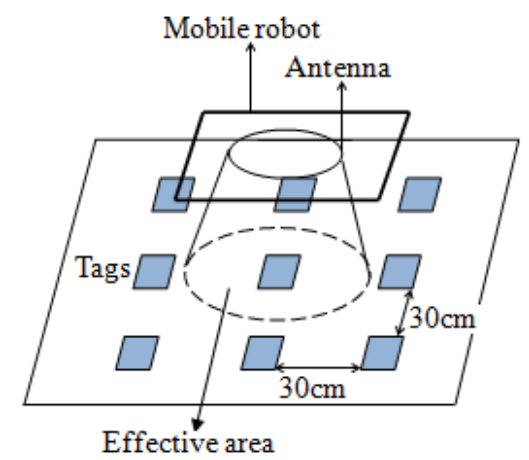

Fig. 3. Sensing area of RFID reader with antenna embedded in mobile robot.

\section{Pathfinding Approach}

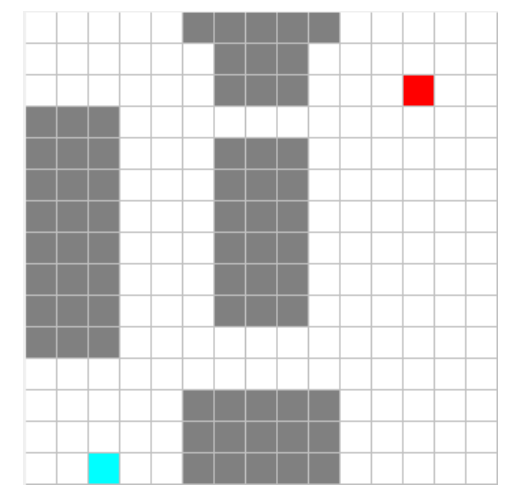

Fig. 4. The virtual map from Fig. 2 and each grid-cell is equal to a passive tag. Blue and red points respectively represent start and destination sites, and gray cells mean there's existence of obstacles.

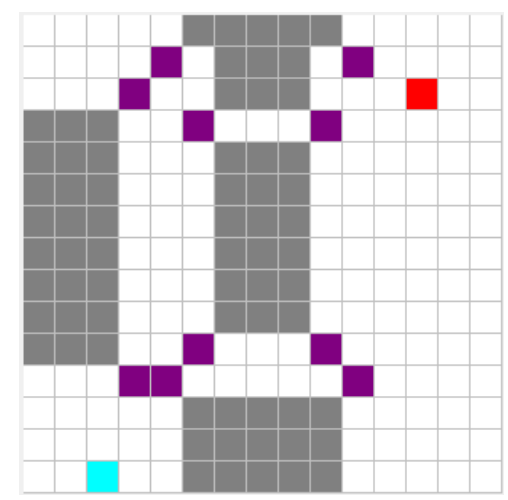

Fig. 5. The grown vertices with purple grid-cells represent feasibly collision-free points from extracting obstacles.
Since we have constructed RFID pattern for offering each tag's position information, we can transfer these data to grid cells for assisting path-planning. We defines that each tag's information is corresponded to each grid cell and the pinned tags are belonged to unwalkable area. As an example, Fig. 4 is a virtual map includes interior information of grid cells from Fig. 1. In our proposed pathfinding approach, we first acquire the outer vertices of obstacle because there's collision in obstacle's corner if we only get the vertices on the spot. Fig. 5 shows the grown vertices from obstacles that don't cause collided occurrence while the mobile robot passes through these corners. Next stage, we utilize heuristic function to find a path in combination of these grown vertices. Therefore, we briefly describe the principle of $\mathrm{A}^{*}$ algorithm.

$A^{*}$ is a well-known search algorithm that can be used for pathfinding. In general, the explored route from the designated starting point can be regarded as four directions or eight directions to search moves, which respectively is called as Manhattan or Euclidean approach. Also, there is an adaption of heuristic estimation acted as the cord of $\mathrm{A}^{*}$ into exploring the accessible path, that can be seen in (3). In the explanation of heuristic estimation, $g(n)$ represents the movement cost from the starting point to any given point $n$, and $h(n)$ stands for the estimated cost from given point $n$ to destination by way of distance calculation. Due to heuristic is an admissible function, $A^{*}$ is guaranteed to find a path from start to goal if there exists a path.

$$
f(n)=g(n)+h(n)
$$

When heuristic estimate starts, in the searching of open list may own some nodes which are not only the lowest $h(n)$ but part of those vertices, then they are recorded as special nodes. They are deemed as turning points/relay points from start on the way to destination if these special nodes finally exist in closed list. Obviously, we unnecessarily care about else nodes in closed list which are not satisfying dual characters. Therefore, there's less redundant navigating information to feedback to mobile robot. The return information includes every steering angle, RFID serial number and moving distance to another relay point. Depending on presetting RFID-based localization model, we can make sure the mobile robot if arrives correct position or not.

\section{IMPLEMENTATION AND RESULTS}

We validated our proposed pathfinding approach by proof-of-concept grid cells which was used to examine feasibility. In the implemented map, a grid cell represents a tag with unique ID and coordinate. First, we considered that there's no existence of obstacles in the map and compared with Manhattan and Euclidean approaches. From Fig. 6 we could find out both approaches did not go straight even without obstacles between starting point and goal because of their directional presetting in example of eight or four directions. Although these settings made more regularity, their paths only moved on $90^{\circ}$ or $45^{\circ}$ which causes to veer 
incessantly. Moreover, there's a known problem that 8-diretional heuristic estimation could just pass through the obstacle corner, but maybe exists collided occurrence in practical navigation, shown as Fig. 7 which includes the comparison of 8-directinal manner and our methods.

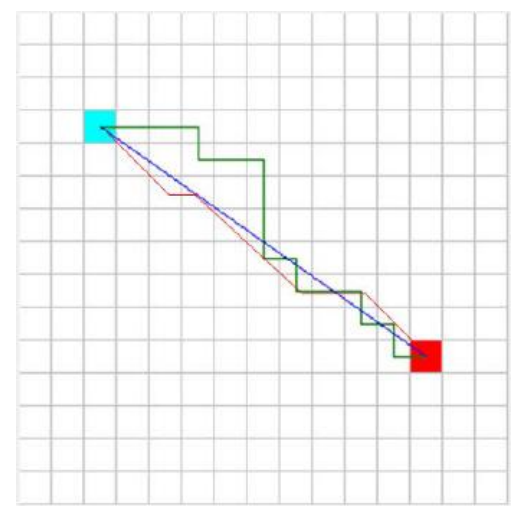

Fig. 6. The path planning without obstacles in comparison of Manhattan (green line), Euclidean (red line) and our proposed approaches (blue line).

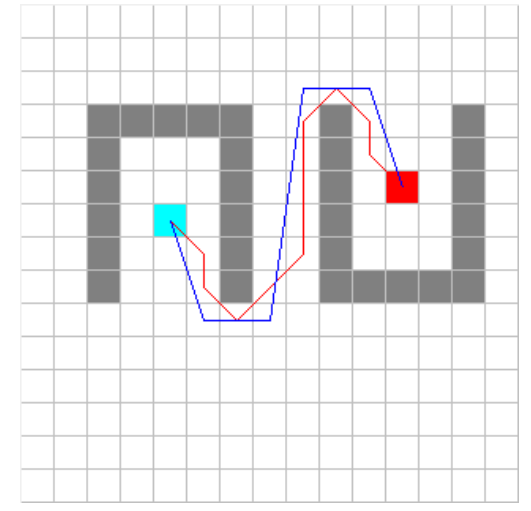

Fig. 7. The capability of obstacles avoidance in our approach but there's occurrence in Euclidean approach (red line).

Beforehand, we established an indoor environment of Fig. 2 includes arrangement of furnishing inside, such as chairs and cabinet looked on as obstacles and estimated costs of those tags under obstacles were infinity. Its virtual achievement was shown as Fig. 8. In the situation, our approach was more efficiency than Manhattan estimate. Furthermore, we test to search larger range and in the complicated circumstance, the Manhattan had to turn many times to arrive goal that would waste lots of time to confirm location and consume power energy, as shown in Fig. 9.

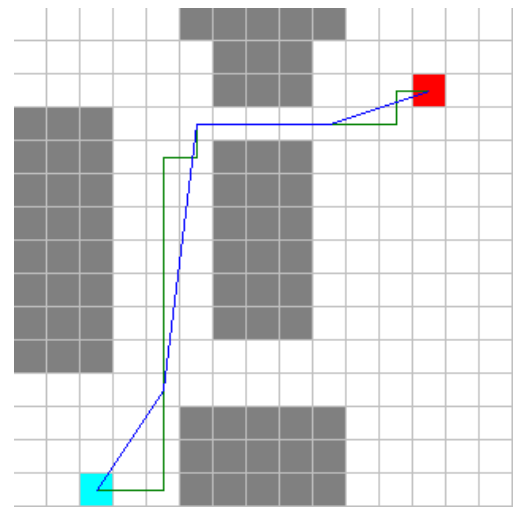

Fig. 8. The simulation of simple environment of Fig. 2. The green line is planned by Manhattan and red line is our revised route.

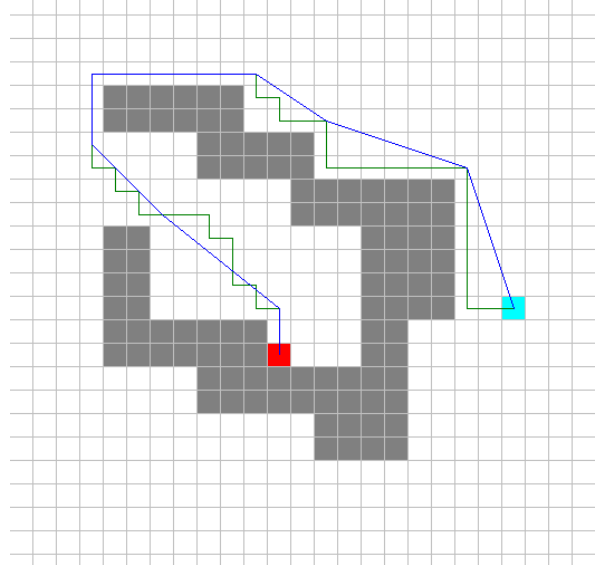

Fig. 9. Lots of veers is planned by Manhattan approach which is not suitable for robot's motion (green line); instead, our method shows improvement our pathfinding algorithm.

\section{CONCLUSIONS}

This paper proposed a minimum-cost pathfinding algorithm based on heuristic estimation with obstacles avoidance capability for helping mobile robot navigation from a starting point to designated destination. The main purpose not only offers a suited path but improves power consumption in real cruise. Therefore, this scheme overcomes the shortcomings and solves too much veering changes compared to conventional $\mathrm{A}^{*}$ algorithm. The path is planned attaches required navigating information, such as the IDs of cruising relay point (assigned FRID tags) and steering angle at any relay point to instruct the movement of mobile robot. In real navigating process, it causes a lot of time waste and power consumption if there are too much relay points. On the contrary, $A^{*}$ approach may not suitable for navigating use because it needs to perform each points of a planned path. Eventually, in positioning confirmation of mobile robot we utilize Cartesian RFID girds to lucidly acquire its approximate location in the period of movement to the destination point.

\section{REFERENCES}

[1] E. W. Dijkstra, "A note on two problems in connexion with graphs," Numerische Mathematik, vol. 1, no.1, pp. 269-271, Dec. 1959.

[2] T. Robert, "Depth-First Search and Linear Graph Algorithms," SIAM Journal on Computing, vol. 1, no. 2, pp. 146-160, June 1972.

[3] S. J. Russell and P. Norvig, Artificial Intelligence: A Modern Approac, 2nd ed., Pearson Education, 2003.

[4] J. Pearl, Heuristics: intelligent search strategies for computer problem solving, Addison-Wesley Longman Publishing Co., 1984.

[5] M. Nakamiya, Y. Kishino, T. Terada, and S. Nishio, "A Route Planning Method Using Cost Map for Mobile Sensor Nodes," in Wireless Pervasive Computing, ISWPC '07. 2nd International Symposium on. 2007.

[6] T. Lozano-Pérez and M. A. Wesley, "An algorithm for planning collision-free paths among polyhedral obstacles," Commun. ACM, vol. 22 , no. 10 , pp. $560-570$, Oct. 1979.

[7] J. Barraquand and J.-C. Latombe, "Robot motion planning: a distributed representation approach," Int. J. Rob. Res., vol. 10, no. 6, pp. 628-649, 1991.

[8] H. Soonshin, L. HyungSoo, and L. JangMyung, "An Efficient Localization Scheme for a Differential-Driving Mobile Robot Based on RFID System," Industrial Electronics, IEEE Transactions on, vol. 54, no. 6, pp. 3362-3369, Dec. 2007.

[9] P. Sunhong, R. Saegusa, and S. Hashimoto, "Autonomous navigation of a mobile robot based on passive RFID," in Proc. the 16th IEEE 
International Symposium on Robot and Human interactive Communication, 2007, pp. 218-223.

[10] P. Sunhong and H. Shuji, "Indoor localization for autonomous mobile robot based on passive RFID," in Proc. IEEE International Conference on Robotics and Biomimetics, 2009, pp. 1856-1861.

[11] B.-S. Choi, J.-W. Lee, J.-J. Lee, and K.-T. Park, "A Hierarchical Algorithm for Indoor Mobile Robot Localization Using RFID Sensor Fusion," Industrial Electronics, IEEE Transactions on, vol. 58, no. 6, pp. 2226-2235, June 2011.

[12] A. A. N. Shirehjini, A. Yassine, and S. Shirmohammadi, "An RFID-Based Position and Orientation Measurement System for Mobile Objects in Intelligent Environments," IEEE Transactions on Instrumentation and Measurement, vol. 61, no. 6, pp. 1664-1675, June 2012.

[13] D. Hahnel, W. Burgard, D. Fox, K. Fishkin, and M. Philipose, "Mapping and localization with RFID technology," in Proc. IEEE International Conference on obotics and Automation, 2004, pp. 1015-1020.

[14] W. Gueaieb and M. S. Miah, "An Intelligent Mobile Robot Navigation Technique Using RFID Technology," IEEE Transactions on Instrumentation and Measurement, vol. 57, no. 9, pp. 1908-1917, Sept. 2008.

[15] L. Catarinucci, S. Tedesco, and L. Tarricone, "Customized Ultra High Frequency Radio Frequency Identification Tags and Reader Antennas Enabling Reliable Mobile Robot Navigation," IEEE Sensors Journal, vol. 13, no. 2, pp. 783-791, Feb. 2013.

[16] D. Ferguson and A. T. Stentz, "Using Interpolation to Improve Path Planning: The Field D* Algorithm," Journal of Field Robotics, vol. 23, no. 2, pp. 79-101, Feb. 2006.

[17] P. Bhattacharya and M. L. Gavrilova, "Roadmap-Based Path Planning - Using the Voronoi Diagram for a Clearance-Based Shortest Path," Robotics \& Automation Magazine, IEEE, vol. 15, no. 2, pp. 58-66, June 2008.

[18] J.-H. Zhou and H.-Y. Lin, "A self-localization and path planning technique for mobile robot navigation," in Proc. 2011 9th World Congress on Intelligent Control and Automation (WCICA), 2011, pp. 694-699.

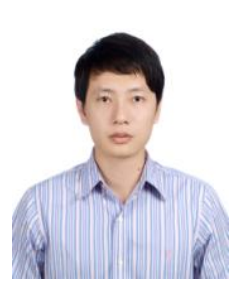

Yung-Fu Hsu graduated with honor from Department of Biomedical Engineering, Chung Yuan Christian University, Chungli, Taiwan ROC in 2009. He received his M.Sc. degree, also with honor, from Institute of Biomedical Engineering, Chung Yuan Christian University, Chungli, Taiwan ROC in 2011. $\mathrm{He}$ is currently a Ph.D. student in Institute of Biomedical Engineering, National
Yang Ming University. His thesis topic involves the deployment of RFID in healthcare and positioning applications.

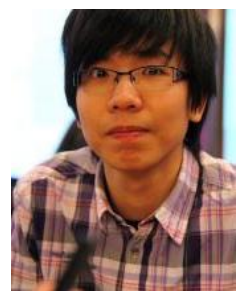

Chun-Hao Huang graduated with honor from Department of Biomedical Engineering, Ming Chuan University, Taoyuan, Taiwan ROC in 2010. He received his M.Sc. degree, also with honor, from Institute of Biomedical Engineering, National Tang Ming University, Taipei, Taiwan ROC in 2012. His thesis topic involves the deployment of RFID in healthcare and positioning applications.

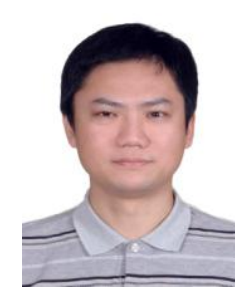

Way-Ren Huang is currently a Ph.D. candidate in Institute of Biomedical Engineering, National Yang Ming University. His thesis topic involves the deployment of RFID in healthcare and health information system which deployed in Taiwan's hospital. The author had rich operation software application in hospital during his Ph.D. education degree. $\mathrm{He}$ is a manager member and teaches data-base structure in TNIA-Taiwan ROC.

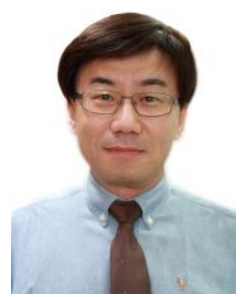

Woei-Chyn Chu is a professor in the Institute of Biomedical Engineering, National Yang Ming University, Taipei, Taiwan ROC. He received his Ph.D. degree in electrical and computer engineering from University of California at Irvine in 1991. He was guest associate editor for Medical Physics and participated in the review/ editorial/ chair work for a number of international journals and conferences. He has published more than 150 research papers and conference presentations in the areas of magnetic resonance imagingquantitation/processing, radiation dosimetry and medical informatics in healthcare.Hereceived four patents in integrating RFID technologies in clinical applications and in accurate RFID real-time tracking. He also obtained several patents in developing medical devices/methodologies to aid orthopedic surgeries and to assist personal homecare management. Currently he has more than 10 patent applications under review. He is member of IEEE-EMBS, ISMRM, BME-Taiwan ROC, MISAT-Taiwan ROC, TAMI-Taiwan ROC and a founding member of Biophysical Society-Taiwan ROC. 